\title{
Inverse role of distinct subsets and distribution of macrophage in lung cancer prognosis: a meta-analysis
}

\author{
Pin Wu ${ }^{1,4, *}$, Dang $\mathbf{W u}^{2,4, *}$, Lufeng Zhao ${ }^{1}$, Lijian Huang ${ }^{1}$, Gang Chen ${ }^{1}$, Gang Shen ${ }^{1}$, \\ Jian Huang ${ }^{3,4}$, Ying Chai ${ }^{1}$ \\ ${ }^{1}$ Department of Thoracic Surgery, Second Affiliated Hospital, Zhejiang University School of Medicine, Zhejiang University, \\ Hangzhou, 310009, China \\ ${ }^{2}$ Department of Radiation Oncology, Second Affiliated Hospital, Zhejiang University School of Medicine, Zhejiang University, \\ Hangzhou, 310009, China \\ ${ }^{3}$ Department of Surgical Oncology, Second Affiliated Hospital, Zhejiang University School of Medicine, Zhejiang University, \\ Hangzhou, 310009, China \\ ${ }^{4}$ Cancer Institute, Second Affiliated Hospital, Zhejiang University School of Medicine, Zhejiang University, Hangzhou, 310009, \\ China \\ *These authors have contributed equally to this work \\ Correspondence to: Pin Wu, email: doctorwp@163.com \\ Ying Chai, email: chaiy@126.com \\ Keywords: tumor-associated macrophage, lung cancer, prognosis, overall survival, meta-analysis \\ Received: November 08, $2015 \quad$ Accepted: May 02, $2016 \quad$ Published: May 26, 2016
}

\section{ABSTRACT}

Background: Tumor-associated macrophages (TAMs) play a crucial role in the regulation of local inflammatory and immune response of tumor microenvironment, being associated with worse outcome of several solid tumors. But the prognostic value of tumor-infiltrating TAMs in lung cancer is still controversial.

Methods: We conduct a meta-analysis of $\mathbf{3 0 5 5}$ patients in 21 studies searched from PubMed and Medline to investigate the correlation between tumor-infiltrating TAMs, including distinct TAM subsets and tissue distribution, and survival of lung cancer. Survival data were computed into odds ratios (ORs) and pooled using MantelHaenszel random-effect model. All statistical tests were two-sided.

Results: High density of tumor-infiltrating TAMs was significantly associated with worse overall survival $(O S)$ at 3 years $(O R=2.45,95 \% C I=1.25$ to $4.80, P=0.009$ ) and 5 years $(O R=2.04,95 \% C I=1.03$ to $4.01, P=0.04)$ of lung cancer. Results for disease free survival (DFS) were similar. M2 subset was associated with worse 3 year-OS and 5 year-OS, whereas M1 subset was associated with better 3-year OS and 5 -year OS. Elevated TAM density in tumor stroma was associated with worse OS at 3 years and 5 years, while elevated TAMs in tumor islet/tumor stroma were associated with better OS at 3 years and 5 years.

Conclusions: Increased tumor-infiltrating TAMs are associated with poor prognosis of lung cancer. M2 subset and TAMs in tumor stroma were associated with worse survival, while M1 subset and TAMs in tumor islet were associated with favorable survival of lung cancer.

\section{INTRODUCTION}

Tumor-associated macrophages (TAMs) play a crucial role in the local inflammatory response and immunosurveillance of cancer $[1,2]$. Plentiful studies have demonstrated that tumor-infiltrating TAMs are associated with worse outcome of human lung cancer [310]. However, other studies reported that tumor-infiltrating
TAMs were associated with favorable outcome of human lung cancer $[11,12]$. It is reported that the type, functional orientation, density, and location of immune cells within distinct tumor regions are all associated with cancer patient survival [13]. Therefore, the divergence maybe due to both the heterogeneity of TAM subsets and functional plasticity of TAMs in local tumor microenvironment $[14,15]$. 
TAMs are generally characterized by the expression of cell surface marker CD68. However, the results from studies evaluating the correlation between TAMs and survival of lung cancer using CD68 as a marker of TAMs are still contradictory $[5,7,9,10,16-20]$. Recently, accumulating studies subdivided TAMs into the classically activated M1 phenotype and the alternatively activated M2 phenotype with an opposite function in tumor immunity [21]. It is reported that tumor-infiltrating M1 is associated with favorable outcome of human lung cancer [11]. On the contrary, some studies suggested that tumor-infiltrating M2 was associated with worse outcome of human lung cancer $[8,9,11,12,18$, 22]. Moreover, TAMs in tumor nest were reported to be associated with better outcome of human lung cancer $[5,11$, $16-18,20]$. Other studies showed that increased TAMs in tumor stroma were negatively correlated with the survival of lung cancer [5, 11, 16-20, 22].

Thus, further studies are needed to clarify the role of tumor-infiltrating TAMs, subsets and intratumoral distribution of TAMs in prognostic prediction of lung cancer. We therefore conducted an exhaustive metaanalysis combining evidence to evaluate the prognostic value of TAMs in human lung cancer. This metaanalysis came into a conclusion that elevated TAMs were associated with worse survival of lung cancer, especially M2 phenotype and TAMs distributing in tumor stroma. While elevated M1 phenotype and TAMs distributing in tumor islet were associated with better survival of lung cancer. Our study suggests that the subsets and tissue distribution of TAMs are very meaningful in prognostic prediction of lung cancer.

\section{RESULTS}

\section{Search results and study characteristics}

Literature searches yield 2338 records and the results are shown in Supplementary Figure S1. The potentially relevant articles were screened for eligibility by duplication, language, abstract and article type, and 1436 records were excluded. Next, 692 citations were excluded for detailed evaluation and at last 21 studies with survival data were included. Characteristics of studies including OS or DFS data are shown in Table 1. A total of 3055 patients were included in those studies.

\section{Evaluation and density of TAM}

A description of the antibodies, detection and definition method of TAM density used in the included studies is shown in Table 1. Various markers were used for the evaluation of TAM density. Seven studies used CD68 antibody, three studies used CD204, one study used CD68 and CD206, one study used CD68 and HLA-DR or CD68 and CD163, one study used TREM-1, one study used IL10 and CD68 and one study used osteopontin and CD68.

\section{Association of TAM with survival of lung cancer}

Our study showed that elevated density of tumorinfiltrating TAMs was associated with worse 3-year OS $(\mathrm{OR}=2.45,95 \%$ confidence interval $(\mathrm{CI})=1.25$ to 4.80 , $\mathrm{P}=0.009)$ (Figure 1A) and 5-year OS $(\mathrm{OR}=2.04,95 \%$ $\mathrm{CI}=1.03$ to $4.01, \mathrm{P}=0.04$ ) (Figure $1 \mathrm{~B}$ ) of lung cancer. Regarding to DFS, high density of tumor-infiltrating TAMs was also associated with worse 3-year DFS (OR $=2.95,95 \% \mathrm{CI}=1.74$ to $5.00, \mathrm{P}<0.0001)($ Figure $2 \mathrm{~A})$ and 5 year DFS $(\mathrm{OR}=2.28,95 \% \mathrm{CI}=1.44$ to $3.60, \mathrm{P}=$ 0.0004 ) (Figure 2B) of lung cancer.

Regarding to the subsets of TAMs, subgroup metaanalysis showed that elevated density of tumor-infiltrating M1 was associated with favorable 3-year OS of lung cancer $(\mathrm{OR}=0.16,95 \% \mathrm{CI}=0.06$ to $0.40, \mathrm{P}=0.0001)$ (Figure 3A). In contrast, elevated density of tumorinfiltrating M2 was associated with worse 3-year OS of lung cancer $(\mathrm{OR}=1.82,95 \% \mathrm{CI}=1.14$ to $2.92, \mathrm{P}=0.01)$ (Figure 3B). Similar to the results of 3-year OS, elevated density of tumor-infiltrating M1 was associated with favorable 5 -year $\mathrm{OS}$ of lung cancer $(\mathrm{OR}=0.19,95 \% \mathrm{CI}=$ 0.08 to $0.43, \mathrm{P}<0.0001$ ) (Figure $4 \mathrm{~A}$ ). In contrast, elevated density of tumor-infiltrating M2 was associated with worse 5 -year OS of lung cancer $(\mathrm{OR}=1.70,95 \% \mathrm{CI}=1.17$ to $2.47, \mathrm{P}=0.002$ ) (Figure 4B). Further study showed that neither M2 in tumor islet nor tumor stroma was associated with 3-year OS of lung cancer (Supplementary Figure $\mathrm{S} 2 \mathrm{~A})$. However, M2 in tumor stroma was associated with worse 5 -year OS of lung cancer $(\mathrm{OR}=2.13,95 \% \mathrm{CI}=$ 1.13 to $4.00, \mathrm{P}=0.01$ ) (Supplementary Figure $\mathrm{S} 2 \mathrm{~B}$ ) but not M2 in tumor islet.

It is interesting that high density of TAMs in tumor islet was correlated with favorable 3-year OS of lung cancer $(\mathrm{OR}=0.41,95 \% \mathrm{CI}=0.18$ to $0.96, \mathrm{P}=0.04$ ) (Figure 5A), but not 5-year OS of lung cancer (Figure 5B). In contrast, elevated density of TAMs in tumor stroma was associated with worse 3 -year $\mathrm{OS}(\mathrm{OR}=1.90,95 \% \mathrm{CI}=$ 1.17 to $3.08, \mathrm{P}=0.009$ ) (Figure $5 \mathrm{C}$ ) and 5-year $\mathrm{OS}(\mathrm{OR}$ $=1.75,95 \% \mathrm{CI}=1.17$ to $2.61, \mathrm{P}=0.006$ ) (Figure $5 \mathrm{D}$ ) of lung cancer. However, high value of tumor islet/tumor stroma rate was significantly correlated with favorable 3 -year OS (OR $=0.09,95 \% \mathrm{CI}=0.03$ to $0.27, \mathrm{P}<0.0001)$ (Figure 5E) and 5-year OS (OR $=0.11,95 \% \mathrm{CI}=0.06$ to $0.21, \mathrm{P}<0.00001$ ) (Figure 5F) of lung cancer.

We also elicit a subgroup meta-analysis according to tumor stage. Results showed that elevated density of tumor-infiltrating TAM was associated with worse 3-year OS (Supplementary Figure S3A) and 5-year OS (Supplementary Figure S3B) in stage I-III.

\section{Sensitivity analyses}

Removal of the studies that used a non-classical marker of TAM (TREM-1 and CD68 combine with IL-10) did not substantially affect the association between TAM density and worse 3 - or 5-year OS $(\mathrm{OR}=2.31,95 \% \mathrm{CI}=$ 
Table 1: Characteristics of studies included in the meta-analysis

\begin{tabular}{|c|c|c|c|c|c|c|c|c|c|c|}
\hline Ref & $\begin{array}{l}\text { Patient } \\
\text { No. }\end{array}$ & $\begin{array}{c}\text { Age, } \\
\text { median } \\
\text { (range) }\end{array}$ & $\begin{array}{c}\text { Male/ } \\
\text { Female }\end{array}$ & Stage & $\begin{array}{l}\text { Follow-up, } \\
\text { median } \\
\text { (range) }\end{array}$ & Marker & $\begin{array}{c}\text { Tissue } \\
\text { Distribution }\end{array}$ & $\begin{array}{l}\text { Cutoff } \\
\text { value }\end{array}$ & $\begin{array}{l}\text { Antibody } \\
\text { (Clone) }\end{array}$ & $\begin{array}{l}\text { NOS } \\
\text { Score }\end{array}$ \\
\hline \multicolumn{11}{|c|}{ Studies including OS } \\
\hline \multirow[t]{2}{*}{$\begin{array}{l}\text { Carus, A., et } \\
\text { al. (2013) }\end{array}$} & 335 & $\begin{array}{l}\geq 65,200 \\
<65,135\end{array}$ & $194 / 141$ & I-IIIA & NR & CD163 & Tumor islet & $\begin{array}{l}\geq 0.21 \% \text { of } \\
\text { tissue area }\end{array}$ & $\begin{array}{c}\text { Anti- } \\
\text { CD163(EDHu-1) }\end{array}$ & 7 \\
\hline & & & & & & & Tumor stroma & $\begin{array}{l}\geq 2.19 \% \text { of } \\
\text { tissue area }\end{array}$ & & \\
\hline $\begin{array}{l}\text { Chen, J. J., } \\
\text { et al. (2003) }\end{array}$ & 35 & 60.3 & $24 / 11$ & I-IIIA & NR & CD68 & $\begin{array}{l}\text { Tumor islet } \\
\text { and stroma }\end{array}$ & $\begin{array}{c}\geq 162 \\
\text { cells/field } \\
(200 \times)\end{array}$ & Anti-CD68(NR) & 7 \\
\hline \multirow[t]{4}{*}{$\begin{array}{l}\text { Dai, } \\
\text { Fuqiang., et } \\
\text { al. (2010) }\end{array}$} & 99 & $60(37-80)$ & $80 / 19$ & I-IV & 96 & CD68 & $\begin{array}{l}\text { Tumor islet } \\
\text { and stroma }\end{array}$ & $\begin{array}{l}\geq 15 \text { cells/ } \\
\text { field } \\
(400 \times)\end{array}$ & Anti-CD68(KP1) & 8 \\
\hline & & & & & & & Tumor islet & & & \\
\hline & & & & & & & Tumor stroma & & & \\
\hline & & & & & & & $\begin{array}{c}\text { Tumor islet/ } \\
\text { stroma }\end{array}$ & & & \\
\hline \multirow[t]{2}{*}{$\begin{array}{l}\text { Hirayama, } \\
\text { S., et al. } \\
(2012)\end{array}$} & 208 & $69(46-88)$ & $188 / 20$ & I-IIIA & 68.4 & CD204 & Tumor stroma & $\begin{array}{l}\geq 30 \text { cells/ } \\
\text { field }(400 \times)\end{array}$ & $\begin{array}{c}\text { Anti- } \\
\text { CD204(A-E5) }\end{array}$ & 8 \\
\hline & & & & & & & Tumor islet & $\begin{array}{l}\geq 9 \text { cells/ } \\
\text { field } \\
(400 \times)\end{array}$ & & \\
\hline $\begin{array}{l}\text { Ho, C. C., } \\
\text { et al. (2008) }\end{array}$ & 68 & NR & $40 / 28$ & I-III & 41 & TREM-1 & $\begin{array}{l}\text { Tumor islet } \\
\text { and stroma }\end{array}$ & $\begin{array}{l}\geq 15 \text { cells/ } \\
\quad \text { field } \\
(400 \times)\end{array}$ & $\begin{array}{l}\text { Anti-TREM- } \\
\text { 1(AF1278) }\end{array}$ & 7 \\
\hline \multirow[t]{3}{*}{$\begin{array}{l}\text { Kawai, O., } \\
\text { et al. (2008) }\end{array}$} & 199 & $62(39-79)$ & $139 / 60$ & IV & NR & CD68 & $\begin{array}{l}\text { Tumor islet/ } \\
\text { stroma }\end{array}$ & $\geq 1$ & Anti-CD68(NR) & 7 \\
\hline & & & & & & & Tumor islet & $\begin{array}{l}\geq 13 \text { cells/ } \\
\text { field } \\
(400 \times)\end{array}$ & & \\
\hline & & & & & & & Tumor stroma & $\begin{array}{l}\geq 12 \text { cells/ } \\
\text { field } \\
(400 \times)\end{array}$ & & \\
\hline \multirow[t]{2}{*}{$\begin{array}{l}\text { Kim, D. W., } \\
\text { et al. (2008) }\end{array}$} & 144 & 60.4 & $106 / 38$ & IA-IV & NR & CD68 & Tumor stroma & $\begin{array}{c}\geq 233 \\
\text { cells } / \mathrm{mm}^{2} \\
(400 \times)\end{array}$ & $\begin{array}{c}\text { Anti- } \\
\text { CD68(M0876) }\end{array}$ & 7 \\
\hline & & & & & & & Tumor islet & $\begin{array}{c}\geq 28 \text { cells/ } \\
\mathrm{mm}^{2} \\
(400 \times)\end{array}$ & & \\
\hline \multirow[t]{2}{*}{$\begin{array}{l}\text { Li, Y., et al. } \\
(2015)\end{array}$} & 159 & $61(44-77)$ & $109 / 50$ & I-III & $46(2-120)$ & CD68 & $\begin{array}{l}\text { Tumor islet } \\
\text { and stroma }\end{array}$ & NR & $\begin{array}{c}\text { Anti- } \\
\text { CD68(ED1) }\end{array}$ & 8 \\
\hline & & & & & & $\begin{array}{l}\text { Osteopontin/ } \\
\text { CD68 }\end{array}$ & $\begin{array}{l}\text { Tumor islet } \\
\text { and stroma }\end{array}$ & NR & $\begin{array}{l}\text { Anti-OPN(NR); } \\
\text { Anti-CD68(KP1) }\end{array}$ & \\
\hline \multirow[t]{3}{*}{$\begin{array}{l}\text { Ma, J., et al. } \\
(2010)\end{array}$} & 100 & NR & $81 / 19$ & I-IV & Max 96 & $\begin{array}{c}\text { CD68/HLA- } \\
\text { DR }\end{array}$ & $\begin{array}{l}\text { Tumor islet } \\
\text { and stroma }\end{array}$ & NR & $\begin{array}{c}\text { Anti- } \\
\text { CD68(KP1); } \\
\text { Anti-HLA- } \\
\text { DR(LN3) }\end{array}$ & 7 \\
\hline & & & & & & & Tumor islet & NR & & \\
\hline & & & & & & & Tumor stroma & NR & & \\
\hline
\end{tabular}

(Continued) 


\begin{tabular}{|c|c|c|c|c|c|c|c|c|c|c|}
\hline Ref & $\begin{array}{l}\text { Patient } \\
\text { No. }\end{array}$ & $\begin{array}{c}\text { Age, } \\
\text { median } \\
\text { (range) }\end{array}$ & $\begin{array}{c}\text { Male/ } \\
\text { Female }\end{array}$ & Stage & $\begin{array}{l}\text { Follow-up, } \\
\text { median } \\
\text { (range) }\end{array}$ & Marker & $\begin{array}{c}\text { Tissue } \\
\text { Distribution }\end{array}$ & $\begin{array}{l}\text { Cutoff } \\
\text { value }\end{array}$ & $\begin{array}{l}\text { Antibody } \\
\text { (Clone) }\end{array}$ & $\begin{array}{l}\text { NOS } \\
\text { Score }\end{array}$ \\
\hline \multirow{5}{*}{$\begin{array}{l}\text { Ohtaki, Y., } \\
\text { et al. (2010) }\end{array}$} & & & & & & $\begin{array}{l}\text { CD68/ } \\
\text { CD163 }\end{array}$ & $\begin{array}{l}\text { Tumor islet } \\
\text { and stroma }\end{array}$ & NR & $\begin{array}{c}\text { Anti- } \\
\text { CD68(KP1); } \\
\text { Anti- } \\
\text { CD163(10D6) }\end{array}$ & \\
\hline & & & & & & & Tumor islet & NR & & \\
\hline & & & & & & & Tumor stroma & NR & & \\
\hline & 170 & $62(33-85)$ & $85 / 85$ & IA-IIIA & 121.2 & CD68 & Tumor islet & $\begin{array}{c}25 \text { cells/ } \\
\mathrm{mm}^{2} \\
(400 \times)\end{array}$ & Anti-CD68(NR) & 8 \\
\hline & & & & & & CD204 & Tumor stroma & $\begin{array}{c}15 \text { cells/ } \\
\mathrm{mm}^{2} \\
(400 \times)\end{array}$ & $\begin{array}{c}\text { Anti- } \\
\text { CD204(A-E5) }\end{array}$ & \\
\hline $\begin{array}{l}\text { Pei, B.-x., et } \\
\text { al. (2014) }\end{array}$ & 417 & NR & $231 / 186$ & I-III & $43(2-120)$ & CD68 & Tumor stroma & $\begin{array}{l}\text { positive } \\
\text { of CD68+ } \\
\text { cells }\end{array}$ & Anti-CD68(KP1) & 7 \\
\hline $\begin{array}{l}\text { Takahashi, } \\
\text { A., et al. } \\
(2013)\end{array}$ & 115 & $68(22-86)$ & $98 / 17$ & IA-IV & 52.8 & CD204 & $\begin{array}{l}\text { Tumor islet } \\
\text { and stroma }\end{array}$ & $\begin{array}{l}\geq 20 \text { cells/ } \\
\text { field } \\
(400 \times)\end{array}$ & $\begin{array}{c}\text { Anti- } \\
\text { CD204(A-E5) }\end{array}$ & 8 \\
\hline $\begin{array}{l}\text { Takanami, } \\
\text { I., et al. } \\
(1999)\end{array}$ & 113 & $62(30-79)$ & $66 / 47$ & I-IV & NR & CD68 & $\begin{array}{l}\text { Tumor islet } \\
\text { and stroma }\end{array}$ & $\begin{array}{c}\text { Densities }> \\
32\end{array}$ & Anti-CD68(KP1) & 7 \\
\hline \multirow[t]{3}{*}{$\begin{array}{l}\text { Welsh, T. J., } \\
\text { et al. (2005) }\end{array}$} & 162 & NR & NR & I-IV & NR & CD68 & Tumor islet & $\begin{array}{c}\geq 131 \\
\text { cells } / \mathrm{mm}^{2} \\
(200 \times)\end{array}$ & $\begin{array}{c}\text { Anti- } \\
\text { CD68(PGM1) }\end{array}$ & 6 \\
\hline & & & & & & & Tumor stroma & $\begin{array}{c}\geq 174 \\
\text { cells } / \mathrm{mm}^{2} \\
(200 \times)\end{array}$ & & \\
\hline & & & & & & & $\begin{array}{l}\text { Tumor islet/ } \\
\text { stroma }\end{array}$ & $\begin{array}{l}\geq \text { Median } \\
\text { value }\end{array}$ & & \\
\hline $\begin{array}{l}\text { Zeni, E., et } \\
\text { al. (2007) }\end{array}$ & 47 & 63.9 & $43 / 7$ & I-IV & NR & IL-10/CD68 & $\begin{array}{l}\text { Tumor islet } \\
\text { and stroma }\end{array}$ & $\begin{array}{l}\geq 16.3 \% \text { of } \\
\text { tissue area }\end{array}$ & $\begin{array}{c}\text { Anti-IL- } \\
\text { 10(NR); Anti- } \\
\text { CD68(M0814) }\end{array}$ & 7 \\
\hline \multirow[t]{2}{*}{$\begin{array}{l}\text { Zhang, B., } \\
\text { et al. (2011) }\end{array}$} & 65 & NR & $38 / 27$ & I-IV & NR & CD68 & $\begin{array}{l}\text { Tumor islet } \\
\text { and stroma }\end{array}$ & $\begin{array}{c}\geq 102 \\
\text { cells/field } \\
(100 \times)\end{array}$ & Anti-CD68(NR) & 6 \\
\hline & & & & & & $\begin{array}{l}\text { CD68/ } \\
\text { CD206 }\end{array}$ & $\begin{array}{l}\text { Tumor islet } \\
\text { and stroma }\end{array}$ & $\begin{array}{l}\geq 82 \text { cells/ } \\
\text { field } \\
(100 \times)\end{array}$ & & \\
\hline $\begin{array}{l}\text { Zhang, W., } \\
\text { et al. (2013) }\end{array}$ & 67 & $71(45-85)$ & $22 / 27$ & I-III & $\begin{array}{c}53.3 \\
(2.1-201.7)\end{array}$ & CD68 & $\begin{array}{l}\text { Tumor islet } \\
\text { and stroma }\end{array}$ & $\begin{array}{l}\geq 50 \% \text { of } \\
\text { tissue area }\end{array}$ & $\begin{array}{c}\text { Anti- } \\
\text { CD68(514H12) }\end{array}$ & 8 \\
\hline \multicolumn{11}{|c|}{ Studies including DFS } \\
\hline $\begin{array}{l}\text { Chen, J. J., } \\
\text { et al. (2003) }\end{array}$ & 35 & 60.3 & $24 / 11$ & I-IIIA & NR & CD68 & $\begin{array}{l}\text { Tumor islet } \\
\text { and stroma }\end{array}$ & $\begin{array}{c}\geq 162 \\
\text { cells/field } \\
(200 \times)\end{array}$ & Anti-CD68(NR) & 7 \\
\hline \multirow[t]{2}{*}{$\begin{array}{l}\text { Hirayama, } \\
\text { S., et al. } \\
(2012)\end{array}$} & 208 & $69(46-88)$ & $188 / 20$ & I-IIIA & 68.4 & CD204 & Tumor stroma & $\begin{array}{c}\geq 30 \text { cells/ } \\
\text { field } \\
(400 \times)\end{array}$ & $\begin{array}{c}\text { Anti- } \\
\text { CD204(A-E5) }\end{array}$ & 8 \\
\hline & & & & & & & Tumor islet & $\begin{array}{l}\geq 9 \text { cells/ } \\
\text { field } \\
(400 \times)\end{array}$ & & \\
\hline
\end{tabular}

(Continued) 


\begin{tabular}{|c|c|c|c|c|c|c|c|c|c|c|}
\hline Ref & $\begin{array}{l}\text { Patient } \\
\text { No. }\end{array}$ & $\begin{array}{c}\text { Age, } \\
\text { median } \\
\text { (range) }\end{array}$ & $\begin{array}{c}\text { Male/ } \\
\text { Female }\end{array}$ & Stage & $\begin{array}{c}\text { Follow-up, } \\
\text { median } \\
\text { (range) }\end{array}$ & Marker & $\begin{array}{c}\text { Tissue } \\
\text { Distribution }\end{array}$ & $\begin{array}{l}\text { Cutoff } \\
\text { value }\end{array}$ & $\begin{array}{l}\text { Antibody } \\
\text { (Clone) }\end{array}$ & $\begin{array}{l}\text { NOS } \\
\text { Score }\end{array}$ \\
\hline $\begin{array}{l}\text { Ho, C. C., } \\
\text { et al. (2008) }\end{array}$ & 68 & NR & $40 / 28$ & I-III & 41 & TREM-1 & $\begin{array}{l}\text { Tumor islet } \\
\text { and stroma }\end{array}$ & $\begin{array}{c}\geq 15 \text { cells/ } \\
\text { field } \\
(400 \times)\end{array}$ & $\begin{array}{c}\text { Anti-TREM- } \\
\text { 1(AF1278) }\end{array}$ & 7 \\
\hline $\begin{array}{l}\text { Ito, M., et } \\
\text { al. (2012) }\end{array}$ & 304 & NR & $139 / 165$ & I & $87(5-181)$ & CD204 & $\begin{array}{l}\text { Tumor islet } \\
\text { and stroma }\end{array}$ & $\begin{array}{l}\geq 8 \text { cells/ } \\
\text { field } \\
(400 \times)\end{array}$ & $\begin{array}{c}\text { Anti- } \\
\text { CD204(A-E5) }\end{array}$ & 7 \\
\hline $\begin{array}{l}\text { Kaseda, K., } \\
\text { et al. (2013) }\end{array}$ & 41 & NR & NR & I & NR & CD204 & Tumor stroma & NR & $\begin{array}{c}\text { Anti- } \\
\text { CD204(A-E5) }\end{array}$ & 6 \\
\hline \multirow[t]{2}{*}{$\begin{array}{l}\text { Li, Y., et al. } \\
(2015)\end{array}$} & 159 & $61(44-77)$ & $109 / 50$ & I-III & $46(2-120)$ & CD68 & $\begin{array}{l}\text { Tumor islet } \\
\text { and stroma }\end{array}$ & NR & $\begin{array}{c}\text { Anti- } \\
\text { CD68(ED1) }\end{array}$ & 8 \\
\hline & & & & & & $\begin{array}{l}\text { Osteopontin/ } \\
\text { CD68 }\end{array}$ & $\begin{array}{l}\text { Tumor islet } \\
\text { and stroma }\end{array}$ & NR & $\begin{array}{l}\text { Anti-OPN(NR); } \\
\text { Anti-CD68(KP1) }\end{array}$ & \\
\hline $\begin{array}{l}\text { Maeda, R., } \\
\text { et al. (2014) }\end{array}$ & 207 & NR & $94 / 113$ & I & NR & CD204 & $\begin{array}{l}\text { Tumor islet } \\
\text { and stroma }\end{array}$ & $\begin{array}{l}\geq 8 \text { cells/ } \\
\text { field } \\
(400 \times)\end{array}$ & $\begin{array}{c}\text { Anti- } \\
\text { CD204(A-E5) }\end{array}$ & 6 \\
\hline $\begin{array}{l}\text { Pei, B.-X., et } \\
\text { al. (2014) }\end{array}$ & 417 & NR & $231 / 186$ & I-III & $43(2-120)$ & CD68 & Tumor stroma & $\begin{array}{l}\text { positive } \\
\text { of CD68+ } \\
\text { cells }\end{array}$ & Anti-CD68(KP1) & 7 \\
\hline $\begin{array}{l}\text { Takahashi, } \\
\text { A., et al. } \\
\text { (2013) }\end{array}$ & 115 & $68(22-86)$ & $98 / 17$ & IA-IV & 52.8 & CD204 & $\begin{array}{l}\text { Tumor islet } \\
\text { and stroma }\end{array}$ & $\begin{array}{c}\geq 20 \text { cells/ } \\
\quad \text { field } \\
(400 \times)\end{array}$ & $\begin{array}{c}\text { Anti- } \\
\text { CD204(A-E5) }\end{array}$ & 8 \\
\hline
\end{tabular}

NR: Not Reported; DFS: disease-free survival; OS: overall survival.

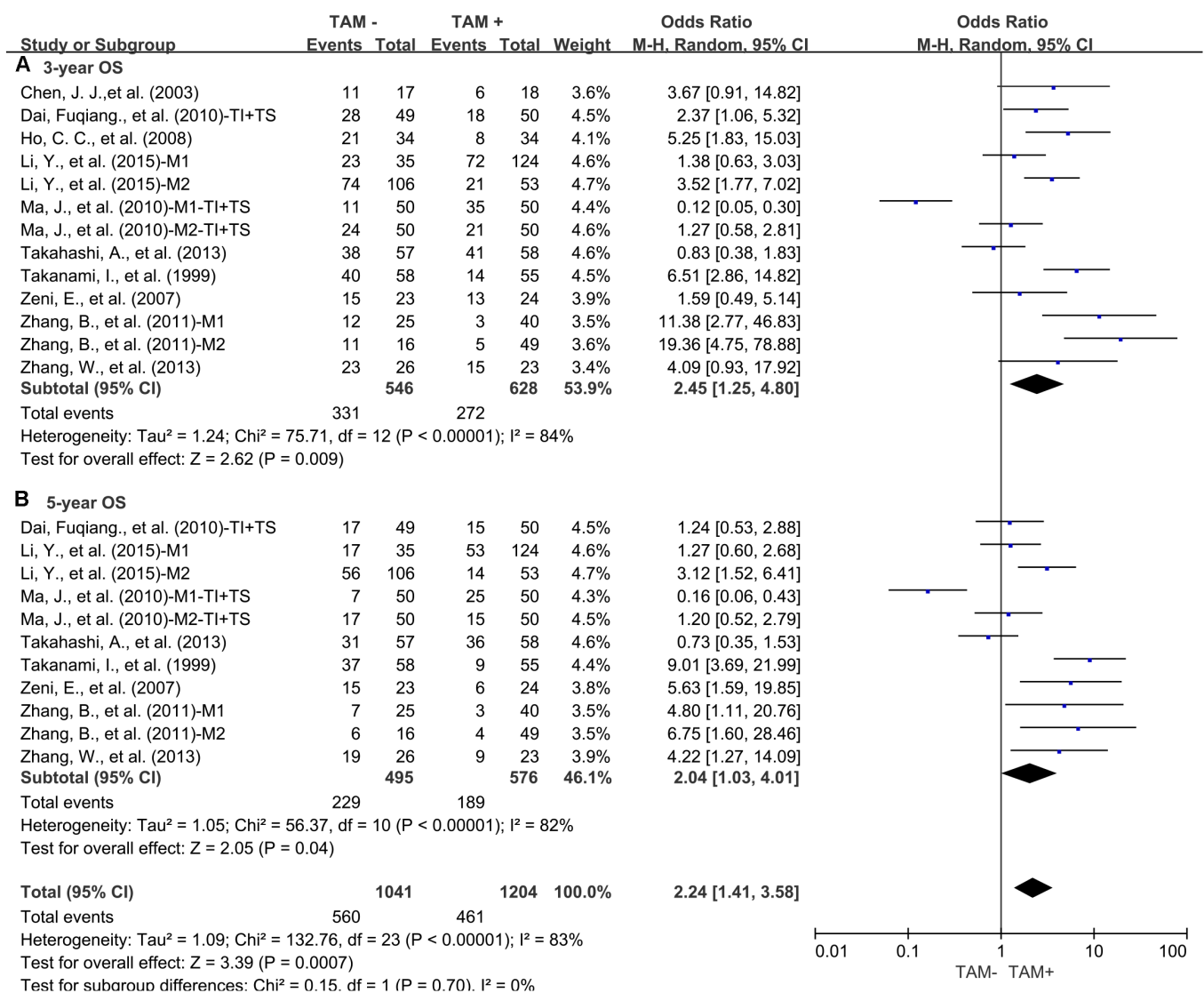

Figure 1: Forest plots showing odds ratios of high density of TAMs versus low density of TAMs for overall survival (OS) at 3 and 5 years. A. 3-year OS; B. 5-year OS. TI: tumor islet; TS: tumor stroma; M1: marker1; M2: marker2. 


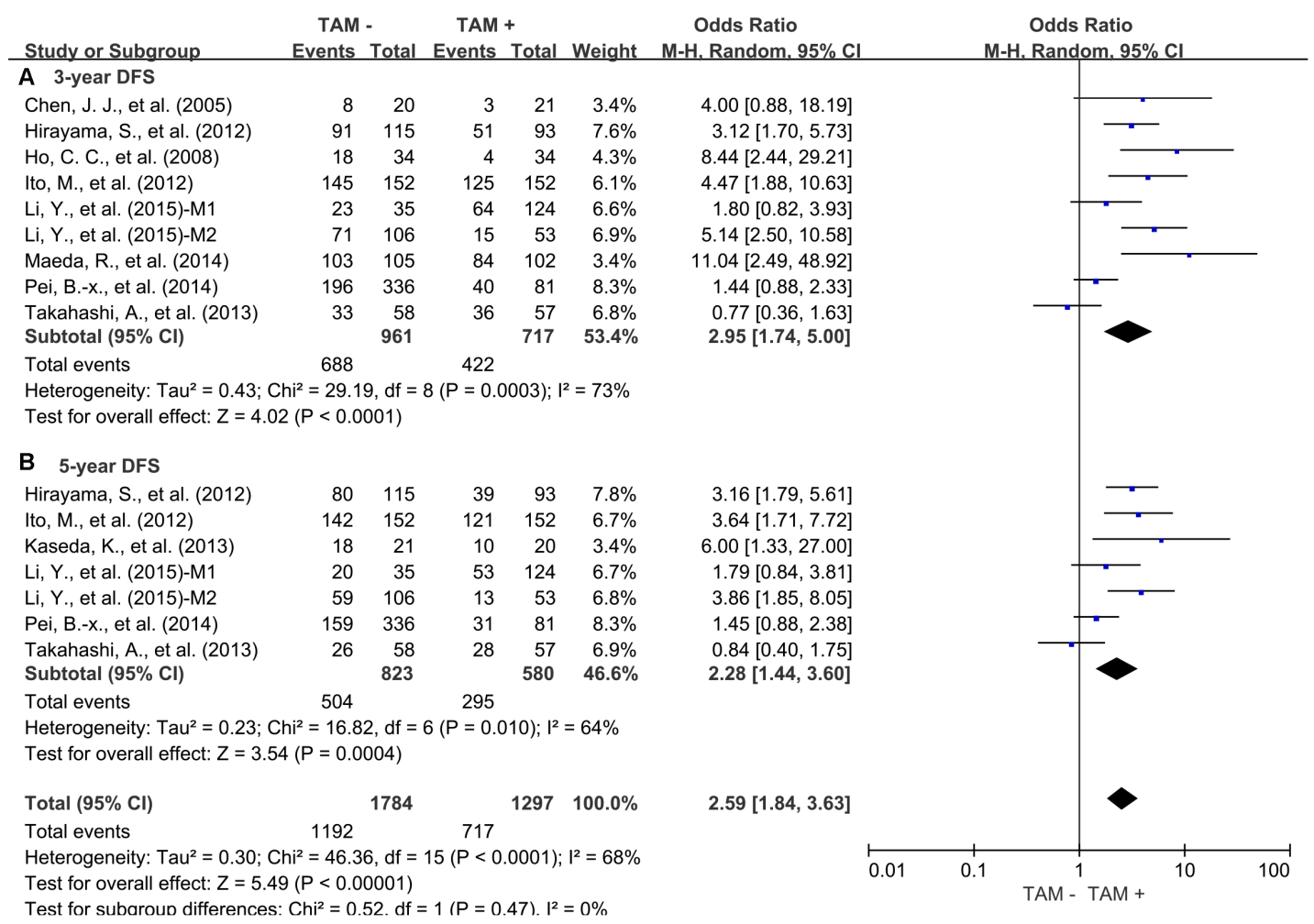

Figure 2: Forest plots showing odds ratios of high density of TAMs versus low density of TAMs for disease free survival (DFS) at 3 and 5 years. A. 3-year DFS; B. 5-year DFS. M1: marker1; M2: marker2.

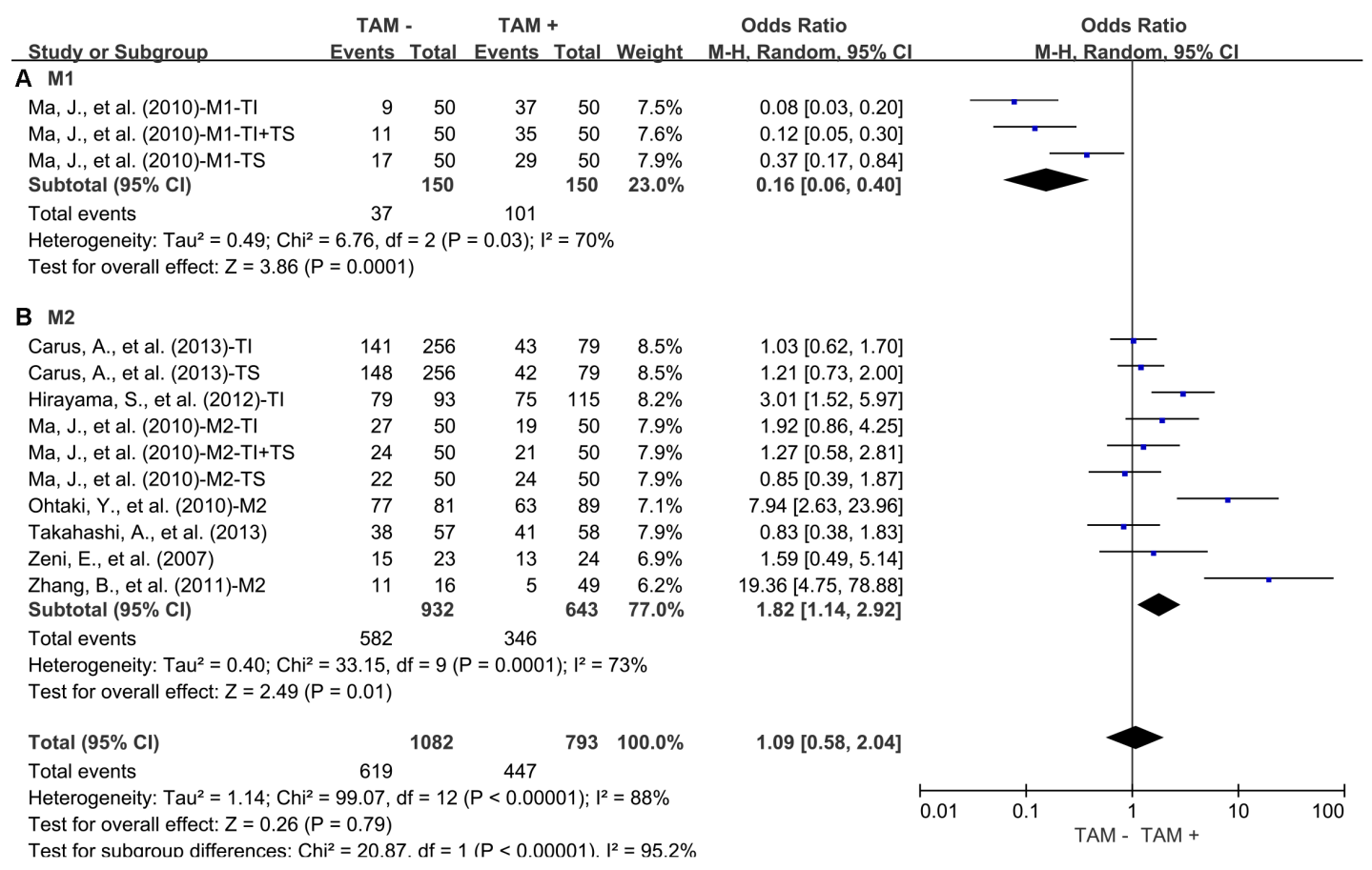

Figure 3: Subgroup analysis of 3-year OS by high density of different TAM subsets. A. M1; B. M2. TI: tumor islet; TS: tumor stroma; M1: CD68 and HLA-DR positive cells; M2: CD163 positive cells, CD204 positive cells, CD68 and CD163 positive cells, CD68 and CD206 positive cells or IL-10 and CD68 positive cells. 


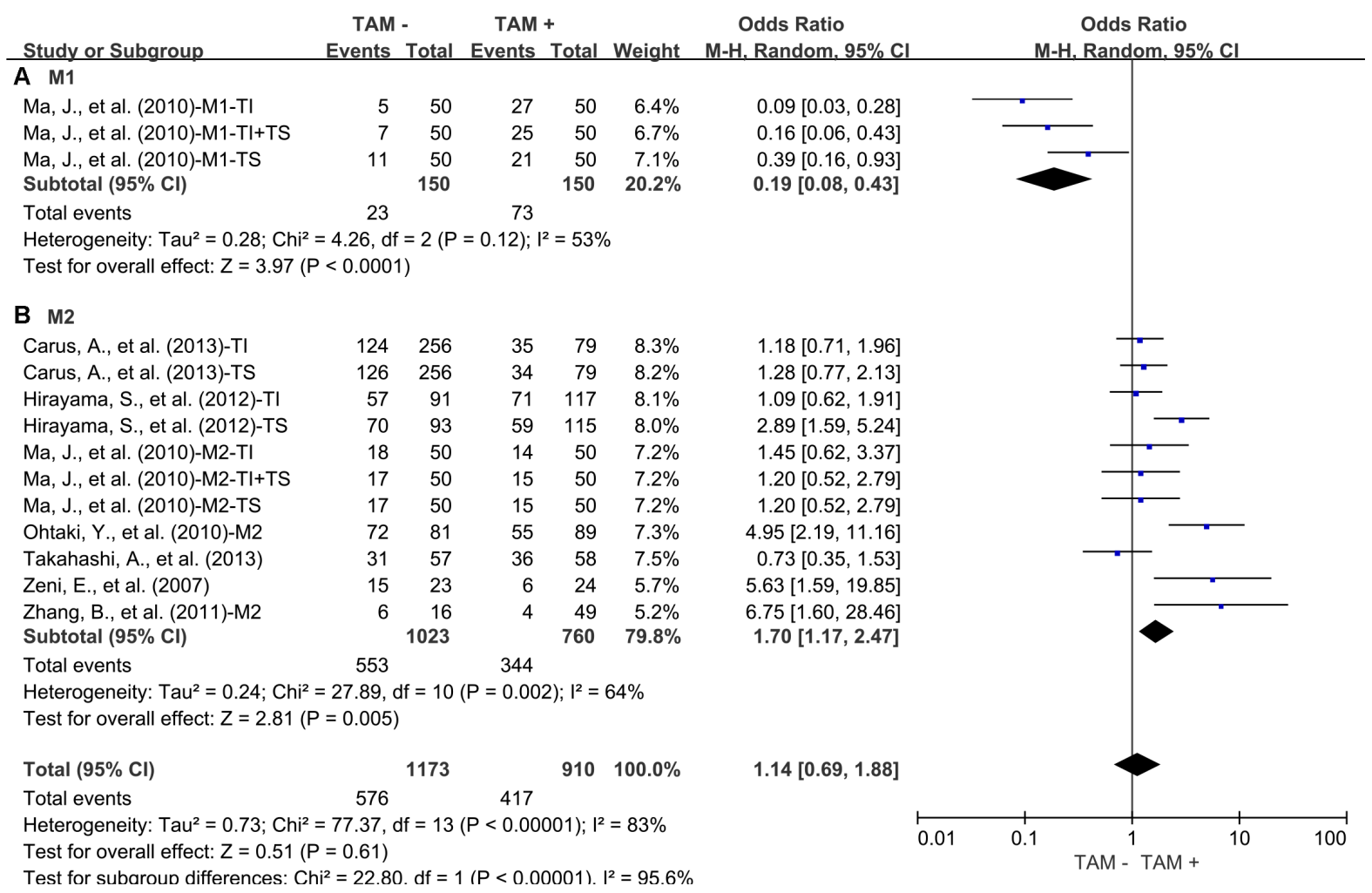

Figure 4: Subgroup analysis of 5-year OS by high density of different TAM subsets. A. M1; B. M2. TI: tumor islet; TS: tumor stroma; M1: CD68 and HLA-DR positive cells; M2: CD163 positive cells, CD204 positive cells, CD68 and CD163 positive cells, CD68 and CD206 positive cells or IL-10 and CD68 positive cells.
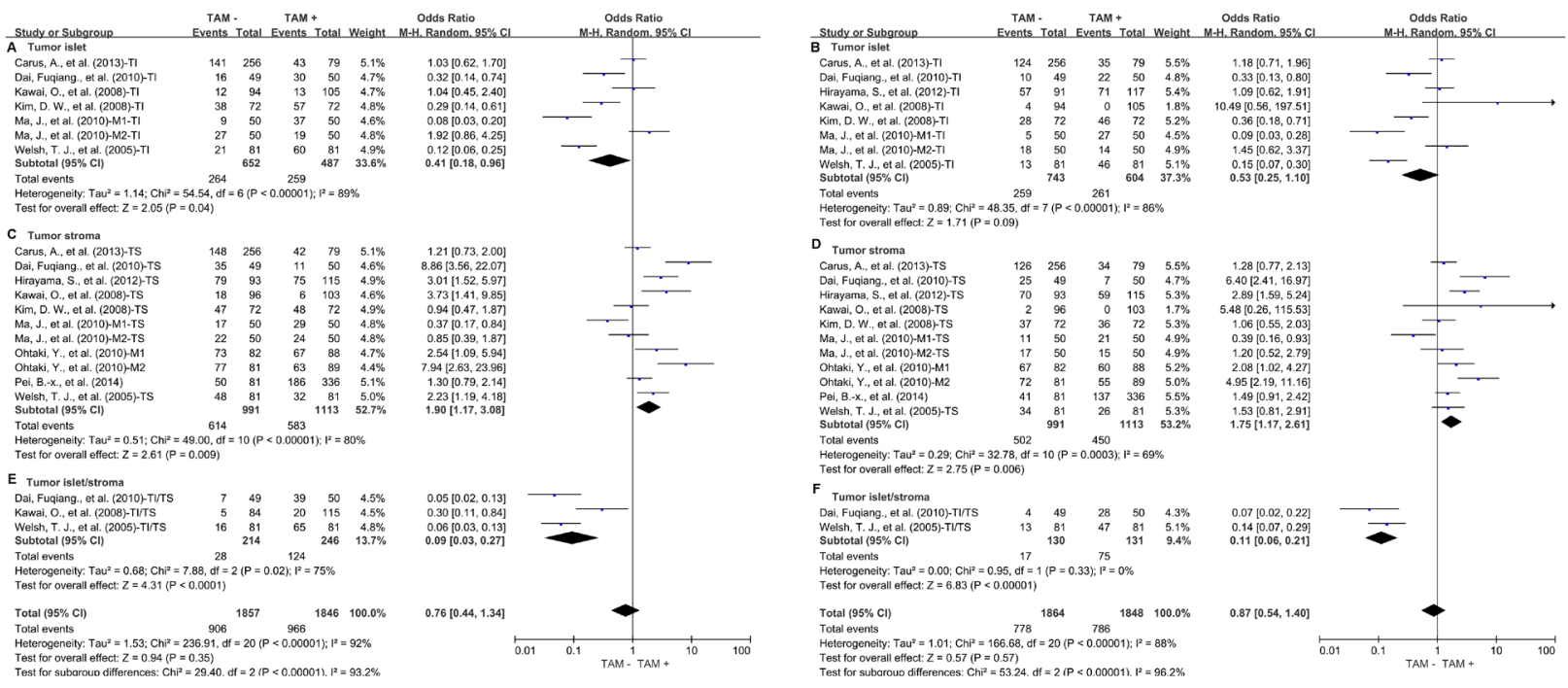

Figure 5: Subgroup analysis of OS by high density of TAMs in different tumor distribution. A. High density of TAMs in TI and 3-year OS; B. High density of TAMs in TI and 5-year OS; C. High density of TAMs in TS and 3-year OS; D. High density of TAMs in TS and 5-year OS; E. High value of TAMs in TI/TS and 3-year OS; F. High value of TAMs in TI/TS and 5-year OS. TI: tumor islet; TS: tumor stroma; M1: marker1; M2: marker2. 
1.13 to $4.68, \mathrm{p}=0.02 ; \mathrm{OR}=2.29,95 \% \mathrm{CI}=1.10$ to 4.74 , $\mathrm{p}=0.03$, respectively). Exclusion of these studies did not reduce heterogeneity for 3- or 5-year OS (Cochran's Q $\mathrm{P}<0.00001, \mathrm{I}^{2}=85 \%$; Cochran's Q P $<0.00001, \mathrm{I}^{2}=$ $82 \%$, respectively). Meta-regression analysis showed that publication year, country, gender and NOS score did not contribute to the heterogeneity (data not shown).

\section{Publication bias}

Funnel plot analysis and Egger's test showed that there was no statistical evidence of publication bias in our meta-analysis.

\section{DISCUSSION}

Numerous studies have demonstrated that TAMs promote cancer initiation and progression via inducing angiogenesis, enhancing tumor cell migration and invasion, and suppressing antitumor immunity [2]. However, the correlation between TAMs and outcome of lung cancer is still under debate. Our comprehensive meta-analysis of 3055 patients included in 21 different studies demonstrates that elevated density of TAMs in tumor is correlated with poor prognosis of lung cancer. However, the correlation between TAMs and outcome of lung cancer is distinct between TAM subsets and intratumoral distribution of TAM. Elevated M2 and TAM in tumor stroma is correlated with poor outcome of lung cancer. In contrast, increased M1 and TAM in tumor nest is correlated with favorable outcome of lung cancer. These findings suggest both the intratumoral distribution and subsets of TAMs are important factors affecting the prognosis of human lung cancer.

Macrophages have traditionally been divided into M1 and M2 subsets and are distinguished by their cell surface markers $[15,23]$. Generally, M1 is suggested to play a crucial role in killing intracellular pathogens and suppressing tumor progression, while M2 can facilitate tumor development in multiple mechanisms [24]. In line with experimental research, our study demonstrates that elevated M1 is correlated with better OS of lung cancer. In contrast, elevated M2 is correlated with worse OS of lung cancer. Further analysis shows that only elevated M2 in tumor stroma is correlated with worse OS of human lung cancer but not in tumor islet. These findings indicate that polarized state of TAM in tumor microenvironment is correlated with clinical outcome of lung cancer patient, and approaches to reprogram TAMs from an M2 to an M1like phenotype are promising in lung cancer therapy.

Accumulated studies reported that there is an inverse association between TAM and prognosis of human lung cancer in tumor islet and tumor stroma $[5,11,16,17$, 20, 25]. Our study shows that elevated CD68+ TAMs in tumor islet are correlated with better OS of lung cancer. In contrast, elevated CD68+ TAMs in tumor stroma are correlated with worse OS of lung cancer. It is interesting that there is a prominent correlation between the ratio of TAMs in tumor islet/tumor stroma and favorable outcome of human lung cancer $[5,16,20]$. However, the correlation between intratumoral distribution of TAM and progress of other human solid tumors are still unclear. Moreover, the discrepant role and underling regulatory mechanisms of TAMs in the different interspace of tumor microenvironment, such as tumor islet and tumor stroma, are needed to be further studied.

This study has several important implications. First, it shows that high TAM density is associated with poor outcome of lung cancer, which suggests that TAMs may be a potential therapeutic target. Second, it suggests the tissue distribution of TAMs plays an important role in tumor progression and prognostic prediction of human lung cancer. Third, it highlights the distinct role of M1 and M2 subsets in tumor progression and prognostic prediction of human lung cancer, which suggests that the key factors involved in M2 polarization may also be a potential therapeutic target.

Some limitations also exist in this meta-analysis. First, the markers and cut-off values for assessing TAMs expression are inconsistent. Second, significant heterogeneity observed among studies cannot be completely interpreted despite the use of appropriate metaanalytic technique with random-effect models. Finally, small studies with negative results may not be published, resulting in publication bias.

In conclusion, our analyses show that elevated density of TAMs in human lung cancer tissues, especially M2 or CD68+ TAMs in tumor stroma, is associated with worse prognosis in human lung cancer, which suggests that directly targeting TAMs or $\mathrm{M} 2$, or reprogramming TAMs from an M2 to an M1phenotype could be promising therapeutic approaches for lung cancer.

\section{MATERIALS AND METHODS}

This meta-analysis was carried out in accordance with preferred reporting items for systematic reviews and meta-analyses statement [26].

\section{Identification and selection of studies}

PubMed and Medline were searched for studies evaluating the density of tumor-infiltrating TAM and survival in lung cancer from 1964 to August 2015. The search terms included "Macrophages" and "Lung Neoplasms" and the results were limited to human studies of lung cancer. We identified a total of 1172 and 1166 entries, respectively. Eligibility criteria were the measurement of tumor-infiltrating TAM by immunohistochemistry (IHC), availability of survival data for at least 3-year survival, and publication in English. Studies Citation lists of retrieved articles were manually 
screened to ensure sensitivity of the search strategy. Study selection was based on the association of the density of TAM in tumor tissue and survival. Inter-reviewer agreement was assessed using Cohen's kappa coefficient. Disagreement was resolved by consensus.

\section{Endpoints of interest}

Overall survival (OS) or disease free survival (DFS) at 3 and 5 years were the primary endpoints. Tumors were classified by TAM density using cut-off as defined by individual studies.

\section{Data collection process}

Two authors (Pin Wu and Dang Wu) independently extracted information using predefined data abstraction forms. The following details were extracted by 2 reviewers (Pin $\mathrm{Wu}$ and Dang $\mathrm{Wu}$ ): number of patients, antibody used for the evaluation, technique used to quantify TAM, and cut-off to determine high density of TAM. Survival data were extracted from tables or Kaplan-Meier curves for both TAM low (control group) and high group (experimental group). The studies included in our meta-analysis were all cohort studies. Two independent authors evaluated the quality of each included study using Newcastle-Ottawa Scale (NOS) [27]. The studies with 6 scores or more were considered as high quality studies. A consensus NOS score for each item was achieved finally.

\section{Data synthesis}

The relative frequency of survival at 3 and 5 years between the control and experimental groups was expressed as an odds ratio (OR) and its 95\% confidence interval (CI). Sensitivity analyses were carried out for different analytical methods and cut-offs for defining the density of TAM and NOS scores for quality assessment of included studies.

\section{Statistical analysis}

Data were extracted from the primary publications and combined into a meta-analysis using RevMan 5.3 analysis software (Cochrane Collaboration, Copenhagen, Denmark). Estimates of ORs were weighted and pooled using the Mantel-Haenszel random effect model. Statistical heterogeneity was assessed using the Cochran's Q and $\mathrm{I}^{2}$ statistics. Differences between subgroups were assessed using methods as previous described by Deeks et al. [28]. Meta-regression analysis was conducted using Stata 12.0 software (StataCorp LP, College Station, TX). All statistical tests were twosided, and statistical significance was defined as $P$ value less than 0.05 . No correction was made for multiple statistical testing.

\section{ACKNOWLEDGMENTS}

The authors thank the members of the laboratory for helpful discussions. This work was supported by grants from the National Natural Science Foundation of China (81572800, PW), the Science and Technology Department of Zhejiang Province (2013c03044-7, YC), and Natural Science Foundation of Zhejiang Province (LY13H160016, YC; Y15H160094, GS and LY15H160041, PW).

\section{CONFLICTS OF INTEREST}

The authors declare no competing financial interest.

\section{REFERENCES}

1. Jaiswal S, Chao MP, Majeti R, Weissman IL. Macrophages as mediators of tumor immunosurveillance. Trends in immunology. 2010; 31:212-219.

2. Pollard JW. Tumour-educated macrophages promote tumour progression and metastasis. Nature reviews Cancer. 2004; 4:71-78.

3. Chen JJ, Lin YC, Yao PL, Yuan A, Chen HY, Shun CT, Tsai MF, Chen CH, Yang PC. Tumor-associated macrophages: the double-edged sword in cancer progression. Journal of clinical oncology. 2005; 23:953-964.

4. Chen JJ, Yao PL, Yuan A, Hong TM, Shun CT, Kuo ML, Lee YC, Yang PC. Up-regulation of tumor interleukin-8 expression by infiltrating macrophages: its correlation with tumor angiogenesis and patient survival in non-small cell lung cancer. Clinical cancer research. 2003; 9:729-737.

5. Dai F, Liu L, Che G, Yu N, Pu Q, Zhang S, Ma J, Ma $\mathrm{L}$, You Z. The number and microlocalization of tumorassociated immune cells are associated with patient's survival time in non-small cell lung cancer. BMC cancer. 2010; 10:220.

6. Ho CC, Liao WY, Wang CY, Lu YH, Huang HY, Chen HY, Chan WK, Chen HW, Yang PC. TREM-1 expression in tumor-associated macrophages and clinical outcome in lung cancer. American journal of respiratory and critical care medicine. 2008; 177:763-770.

7. Takanami I, Takeuchi K, Kodaira S. Tumor-associated macrophage infiltration in pulmonary adenocarcinoma: association with angiogenesis and poor prognosis. Oncology. 1999; 57:138-142.

8. Zeni E, Mazzetti L, Miotto D, Lo Cascio N, Maestrelli P, Querzoli P, Pedriali M, De Rosa E, Fabbri LM, Mapp CE, Boschetto P. Macrophage expression of interleukin-10 is a prognostic factor in nonsmall cell lung cancer. The European respiratory journal. 2007; 30:627-632.

9. Zhang B, Yao G, Zhang Y, Gao J, Yang B, Rao Z, Gao J. M2-polarized tumor-associated macrophages are associated with poor prognoses resulting from accelerated 
lymphangiogenesis in lung adenocarcinoma. Clinics (Sao Paulo, Brazil). 2011; 66:1879-1886.

10. Zhang W, Pal SK, Liu X, Yang C, Allahabadi S, Bhanji S, Figlin RA, Yu H, Reckamp KL. Myeloid clusters are associated with a pro-metastatic environment and poor prognosis in smoking-related early stage non-small cell lung cancer. PloS one. 2013; 8:e65121.

11. Ma J, Liu L, Che G, Yu N, Dai F, You Z. The M1 form of tumor-associated macrophages in non-small cell lung cancer is positively associated with survival time. BMC cancer. 2010; 10:112.

12. Takahashi A, Ishii G, Kinoshita T, Yoshida T, Umemura S, Hishida T, Yoh K, Niho S, Goto K, Ohmatsu H, Ohe Y, Nagai K, Ochiai A. Identification of prognostic immunophenotypic features in cancer stromal cells of high-grade neuroendocrine carcinomas of the lung. Journal of cancer research and clinical oncology. 2013; 139:1869-1878.

13. Galon J, Angell HK, Bedognetti D, Marincola FM. The continuum of cancer immunosurveillance: prognostic, predictive, and mechanistic signatures. Immunity. 2013; 39:11-26.

14. Allavena P, Sica A, Garlanda C, Mantovani A. The YinYang of tumor-associated macrophages in neoplastic progression and immune surveillance. Immunological reviews. 2008; 222:155-161.

15. Biswas SK, Mantovani A. Macrophage plasticity and interaction with lymphocyte subsets: cancer as a paradigm. Nature immunology. 2010; 11:889-896.

16. Kawai O, Ishii G, Kubota K, Murata Y, Naito Y, Mizuno T, Aokage K, Saijo N, Nishiwaki Y, Gemma A, Kudoh $\mathrm{S}$, Ochiai A. Predominant infiltration of macrophages and $\mathrm{CD} 8(+) \mathrm{T}$ Cells in cancer nests is a significant predictor of survival in stage IV nonsmall cell lung cancer. Cancer. 2008; 113:1387-1395.

17. Kim DW, Min HS, Lee KH, Kim YJ, Oh DY, Jeon YK, Lee SH, Im SA, Chung DH, Kim YT, Kim TY, Bang YJ, Sung SW, Kim JH, Heo DS. High tumour islet macrophage infiltration correlates with improved patient survival but not with EGFR mutations, gene copy number or protein expression in resected non-small cell lung cancer. British journal of cancer. 2008; 98:1118-1124.

18. Ohtaki Y, Ishii G, Nagai K, Ashimine S, Kuwata T, Hishida T, Nishimura M, Yoshida J, Takeyoshi I, Ochiai A. Stromal macrophage expressing CD204 is associated with tumor aggressiveness in lung adenocarcinoma. Journal of thoracic oncology. 2010; 5:1507-1515.
19. Pei B-x, Sun B-s, Zhang Z-f, Wang A-1, Ren P. Interstitial tumor-associated macrophages combined with tumorderived colony-stimulating factor- 1 and interleukin-6, a novel prognostic biomarker in non-small cell lung cancer. The Journal of thoracic and cardiovascular surgery. 2014; 148:1208-1216.e1202.

20. Welsh TJ, Green RH, Richardson D, Waller DA, O'Byrne $\mathrm{KJ}$, Bradding P. Macrophage and mast-cell invasion of tumor cell islets confers a marked survival advantage in non-small-cell lung cancer. Journal of clinical oncology. 2005; 23:8959-8967.

21. Mantovani A, Sozzani S, Locati M, Allavena P, Sica A. Macrophage polarization: tumor-associated macrophages as a paradigm for polarized M2 mononuclear phagocytes. Trends in immunology. 2002; 23:549-555.

22. Hirayama S, Ishii G, Nagai K, Ono S, Kojima M, Yamauchi C, Aokage K, Hishida T, Yoshida J, Suzuki K, Ochiai A. Prognostic impact of CD204-positive macrophages in lung squamous cell carcinoma: possible contribution of Cd204-positive macrophages to the tumor-promoting microenvironment. Journal of thoracic oncology. 2012; 7:1790-1797.

23. Gordon S, Martinez FO. Alternative activation of macrophages: mechanism and functions. Immunity. 2010; 32:593-604.

24. Mantovani A, Allavena P. The interaction of anticancer therapies with tumor-associated macrophages. The Journal of experimental medicine. 2015; 212:435-445.

25. Carus A, Ladekarl M, Hager H, Pilegaard H, Nielsen PS, Donskov F. Tumor-associated neutrophils and macrophages in non-small cell lung cancer: no immediate impact on patient outcome. Lung cancer (Amsterdam, Netherlands). 2013; 81:130-137.

26. Liberati A, Altman DG, Tetzlaff J, Mulrow C, Gotzsche PC, Ioannidis JP, Clarke M, Devereaux PJ, Kleijnen J, Moher D. The PRISMA statement for reporting systematic reviews and meta-analyses of studies that evaluate health care interventions: explanation and elaboration. PLoS medicine. 2009; 6:e1000100.

27. Stang A. Critical evaluation of the Newcastle-Ottawa scale for the assessment of the quality of nonrandomized studies in meta-analyses. European journal of epidemiology. 2010; 25:603-605.

28. Deeks JJ HJ, Altman DG. Analysing and presenting results. In: Higgins JPT, Green S, ed. Cochrane Handbook for Systematic Reviews of Interventions 425 Chichester, UK: John Wiley \& Sons. 2006. 